\title{
The Light Cupula: An Emerging New Concept for Positional Vertigo
}

\author{
Min-Beom Kim ${ }^{1}$, Seok Min Hong ${ }^{2}$, Hyerang $\mathrm{Choi}^{3}$, Seongjun Choi ${ }^{3}$, \\ Ngoc Chien Pham ${ }^{3}$, Jung Eun Shin ${ }^{3}$, and Chang-Hee Kim ${ }^{3}$ \\ ${ }^{1}$ Department of Otorhinolaryngology-Head and Neck Surgery, Kangbuk Samsung Hospital, Sungkyunkwan University \\ School of Medicine, Seoul, \\ ${ }^{2}$ Department of Otorhinolaryngology-Head and Neck Surgery, Dongtan Sacred Heart Hospital, Hallym University College of Medicine, \\ Hwaseong, \\ ${ }^{3}$ Department of Otorhinolaryngology-Head and Neck Surgery, Konkuk University Medical Center, Konkuk University \\ School of Medicine, Seoul, Korea
}

Received August 29, 2017

Revised September 12, 2017

Accepted September 14, 2017

\section{Address for correspondence}

Chang-Hee Kim, MD, PhD

Department of Otorhinolaryngology-

Head and Neck Surgery,

Konkuk University Medical Center,

Konkuk University

School of Medicine,

120-1 Neungdong-ro, Gwangjin-gu,

Seoul 05030, Korea

Tel $+82-2-2030-7666$

Fax +82-2-2030-5299

E-mail ryomachang@gmail.com
Benign paroxysmal positional vertigo (BPPV) is the most common type of positional vertigo. A canalolithiasis-type of BPPV involving the lateral semicircular canal (LSCC) shows a characteristic direction-changing positional nystagmus (DCPN) which beats towards the lower ear (geotropic) on turning the head to either side in a supine position. Because geotropic DCPN in LSCC canalolithiasis is transient with a latency of a few seconds, the diagnosis can be challenging if geotropic DCPN is persistent without latency. The concept of "light cupula" has been introduced to explain persistent geotropic DCPN, although the mechanism behind it requires further elucidation. In this review, we describe the characteristics of the nystagmic pattern in light cupula and discuss the current evidence for possible mechanisms explaining the phenomenon.

J Audiol Otol 2018;22(1):1-5

KEY WORDS: Light cupula $\cdot$ Positional vertigo $\cdot$ Head-roll test $\cdot$ Direction-changing positional nystagmus $\cdot$ Null plane.

\section{Introduction}

In Benign paroxysmal positional vertigo involving lateral semicircular canal (LSCC), positional nystagmus is characterized as a direction-changing positional nystagmus (DCPN) on a head-roll test (HRT) in the supine position. When the DCPN beats towards the lower ear (geotropic), a gravity-dependent movement of otolith particles within the LSCC is considered to be the cause (canalolithiasis). Geotropic DCPN in LSCC canalolithiasis is transient; it usually ceases within one minute and can be fatigued. However, patients have been reported with positional vertigo who display a persistent geotropic DCPN, without latency or fatigability. To explain this variant of geotropic DCPN, the "light cupula" mechanism was introduced

This is an Open Access article distributed under the terms of the Creative Commons Attribution Non-Commercial License (http://creativecommons.org/licenses/by-nc/4.0/) which permits unrestricted non-commercial use, distribution, and reproduction in any medium, provided the original work is properly cited.
[1]. Since then, several studies have been reported on light cupula [2-5]. A study demonstrated that the nystagmus was persistent in $14.2 \%$ of patients with geotropic DCPN [3], and recurrence of light cupula was reported to be common on both the same and the opposite side [3,6]. In this review, the characteristics of the nystagmic pattern in light cupula are described, and possible mechanisms explaining the phenomenon are discussed.

\section{Characteristics of Nystagmus}

Both LSCC canalolithiasis and light cupula show geotropic DCPN on a HRT, which makes their differentiation challenging, unless the positional nystagmus is observed for a long time. In the healthy state, semicircular canals (SCCs) are gravity-independent because the specific gravity of the cupula is equal to that of the surrounding endolymph. However, when the cupula becomes lighter (light cupula) or heavier (heavy cupula) 
than the surrounding endolymph, deflection of the cupula may occur under the influence of gravity. Under these circumstances, persistent positional nystagmus can be observed under the influence of gravity.

To understand the characteristics of positional nystagmus in light cupula, knowledge about the orientation of the LSCC plane within the temporal bone and the axis of the cupula within the LSCC is important. The anterior part of the LSCC is tilted upwards, approximately $30^{\circ}$ from the horizontal plane (Fig. 1A), and the LSCC cupula is tilted laterally from the sagittal plane (Fig. 1B). In light cupula, a spontaneous nystagmus, beating towards the unaffected side, is observed in the upright seated position; the nystagmus ceases when the head is bent slightly forward about $30^{\circ}$, placing the LSCC parallel to the horizontal plane and perpendicular to the direction of the gravitational force [6]. Persistent horizontal nystagmus beating towards the affected side and towards the healthy side is observed in bowing and leaning positions, respectively. A HRT elicits persistent geotropic DCPN, and the intensity of nystagmus is stronger on turning the head to the affected side, according to Ewald's second law. When the head is slightly turned to the affected side in the supine position until the LSCC cupula is aligned with the plane of the gravitational vector, the nystagmus stops; the direction changes with further head-turn. This point has been referred to as the neutral point $[1,2]$, zero zone [4,7], neutral position [5,8,9], null plane [3], or null point [6]. The presence of null plane is a crucial finding for differential diagnosis and determination of the laterality of the cupulopathy (light cupula and heavy cupula) [6]. The points for differentiating light cupula from LSCC canalolithiasis are summarized in Table 1.
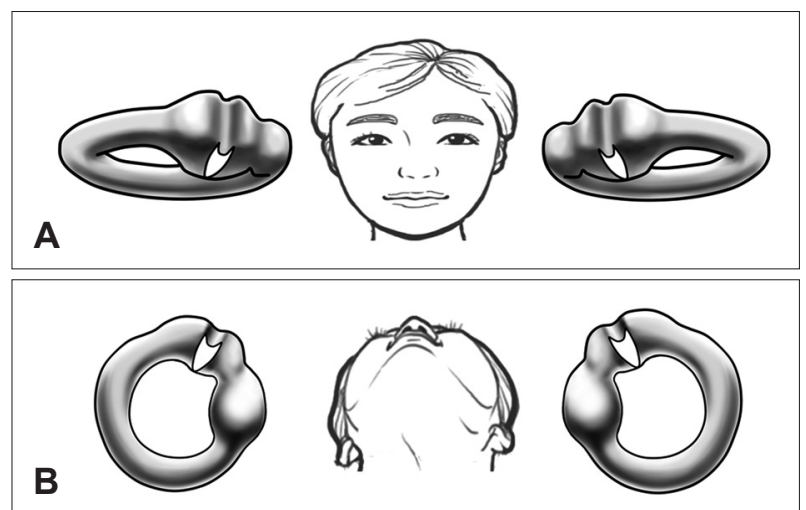

Fig. 1. Anatomical orientation of the LSCC cupula in the temporal bone. A: Frontal view. Magnified images of the both LSCCs are shown; the anterior part of the LSCC can be seen tilted approximately $30^{\circ}$ upwards from the horizontal plane. B: In a supine position, the LSCC cupula is tilted laterally from the sagittal plane. The long axis of the LSCC cupula is oriented in a medial to lateral direction. Adapted from Kim, et al. Laryngoscope 2015;125:19215 [6]. LSCC: lateral semicircular canal.

\section{Pathophysiological Mechanism}

\section{Central vs. peripheral mechanism}

The pathophysiological mechanism behind light cupula remains controversial. It has been reported that geotropic DCPN can be observed in patients with central nervous system disorders. Von Brevern, et al. [10] described three patients with definite vestibular migraine (VM) who showed positional nystagmus during an acute episode. Persistent geotropic DCPN, persistent apogeotropic DCPN, and transient purely torsional nystagmus in both lateral supine positions were reported in one patient each. Polensek and Tusa [11] reported that persistent DCPN was the most commonly observed pattern of positional nystagmus in acute VM patients, though they did not clarify whether it was geotropic or apogeotropic. This central positional nystagmus was usually of a low velocity; it was persistent, lacked latency, and was present only when the patients were examined during a symptomatic migraine spell. Lechner, et al. [12] reported that 5 of 13 acute VM patients exhibited persistent geotropic DCPN which was characteristically symmetrical on both head-rolling and had a low velocity that was different from LSCC canalolithiasis which showed a crescendo-decrescendo pattern with a high velocity. It was suggested that dysfunction of inhibitory GABAergic connections from the vestibulocerebellum to the vestibular nuclei may be the cause of persistent geotropic DCPN in VM patients [10]. Lee, et al. [13] reported two patients who were initially considered to have LSCC canalolithiasis but were ultimately diagnosed with a central lesion. The final diagnoses were cerebellar glioma and lateral medullary infarct, respectively. Yang and Oh [14] reported a patient with human immunodeficiency virus encephalopathy who showed persistent geotropic DCPN. In this patient, spontaneous nystagmus was not observed, DCPN was more intense when the head was turned to the left side than to the right, and nystagmus was not elicited on bowing or leaning. Choi, et al. [15] reported a patient with light cupula caused by meningitis with high cerebrospinal fluid (CSF)

Table 1. Characteristic findings in positional nystagmus to differentiating LSCC canalolithiasis from light cupula

\begin{tabular}{lcc}
\hline & LSCC canalolithiasis & Light cupula \\
\hline Head-roll test & & \\
Direction & Geotropic DCPN & Geotropic DCPN \\
Duration & Transient & Persistent \\
Latency & Yes & No \\
Null plane & No & Yes \\
Fatigability & Yes & No \\
\hline
\end{tabular}

Adapted from Kim, et al. Laryngoscope 2014;124:E15-9 [3]. DCPN: direction-changing positional nystagmus, LSCC: lateral semicircular canal 
protein, and speculated that increased CSF protein concentration elevated the endolymphatic protein content, increasing the specific gravity of the endolymph.

Imai, et al. [5] demonstrated that the time-constant of slowphase velocity in persistent geotropic DCPN is comparable to that of persistent apogeotropic DCPN, and suggested that because the pathophysiological basis of persistent apogeotropic DCPN is considered to be a heavy cupula, persistent geotropic DCPN is conversely induced by a light cupula of peripheral origin. It has been reported that the presence of a null plane is an important clinical characteristics of cupulopathy, considering the orientation of LSCC cupula [6]; when a null plane is identified and neurologic examination reveals no other abnormal findings, cupulopathy of peripheral origin may be the likely cause of persistent geotropic DCPN [1-3,6,8].

\section{"Lighter cupula" theory}

The "lighter cupula" theory originated from the concept of positional alcohol nystagmus (PAN) [16]. It was hypothesized that, after intake, alcohol within the blood stream diffuses into the cupula faster than it does into the surrounding endolymph, which makes the cupula lighter (Phase I of PAN). This theory was further supported by the findings that a heavy cupula could be induced by ingestion of heavy water (deuterium oxide) [17], PAN was not induced in patients with bilateral labyrinthine destruction, and PAN compatible with a unilateral light cupula was reproduced in hemi-labyrinthectomized patients [18]. The sulfated proteoglycans, found in the endolymphatic sac, are reported to be synthesized in the cupula and secreted into the endolymph. Bergenius and Tomanovic [4] suggested that an altered homeostasis of these macromolecules may change the relative specific gravity of the endolymph to the cupula. Seo, et al. [19] reported a patient with sudden sensorineural hearing loss showing persistent geotropic DCPN whose positional vertigo was intractable and persisted for more than 6 months. They suspected that the light cupula in that patient was caused by an irreversible morphological change in the cupula (enlarged cupula). An animal study showed that intra-tympanic injection of solutions with various specific gravities elicited DCPN [20]. Persistent geotropic or apogeotropic DCPN was induced by injection of solutions with lower or higher specific gravity than that of the endolymph, respectively. It was suspected that injected substances passed into the perilymph from the middle ear via the round window membrane and then deposited in the cupula, and this assumption that was supported by the histologic finding that precipitation of eosinophilic materials was observed around the round window membrane and the cupula [20].

\section{"Heavier endolymph" theory}

The "heavier endolymph" theory has been proposed on the assumption that the specific gravity of the endolymph may increase due to an acute insult on the inner ear such as labyrinthine hemorrhage, inner ear hypoperfusion, or inflammation [3]. Hiruma, et al. [2] reported a patient who developed a light cupula after stellate ganglion block; they suggested that increased flow in the vertebral artery after stellate ganglion block may cause inner ear hypoperfusion, leading to a change in the density or viscosity of the endolymph. Kim, et al. $[3,21]$ speculated that blood plasma proteins leaking into the inner ear fluids due to breakage of the blood-labyrinthine barrier may increase the specific gravity of the endolymph, based on the observation that some patients of sudden sensorineural hearing loss with vertigo show persistent geotropic DCPN. This hypothesis was further supported by the conversion between geotropic and apogeotropic persistent DCPN, assumed to be caused by an over-compensation of endolymphatic homeostasis [22].

The "heavier endolymph" theory has been criticized on two counts; 1) an abrupt symptomatic onset and a short clinical course cannot reasonably be explained by a change in specific gravity of the endolymph and 2) LSCC is the only affected SCC in most cases of light cupula [8]. However, it takes about 2 weeks [3] for vertigo or nystagmus to disappear in most patients with light cupula, which is much longer than the natural course of LSCC canalolithiasis or cupulolithiasis without treatment [23]. Moreover, when light cupula is associated with sudden deafness [24] or meningitis [15], vertigo usually develops several hours or days after the onset of hearing loss or the symptoms of meningitis; this is speculated to be because time is required to elevate the specific gravity of the vestibular endolymph. Endolymphatic fluids circulate within the inner ear organs, which are interconnected by the endolymphatic membranes. Thus, if "heavier endolymph" is the underlying mechanism, it is reasonable to assume that the light cupula phenomenon would occur in all 3 SCCs and otolith organs. Though it has recently been demonstrated that light cupula may involve all three SCCs on the affected side [25], LSCC alone was reported to be involved in all previous reports on the phenomenon. The reasons why horizontal nystagmus predominates in light cupula have been speculated as follows; 1) vertical and torsional components of nystagmus may be mixed or canceled due to activation or inhibition of vertical SCCs and 2) the exact alignment of the cupula in the vertical SCCs with respect to the gravity has not been clearly identified [3,25]. Hiruma and Numata [1] suggested that vertical and torsional components of nystagmus might be concealed in light cupula, based on the findings of an animal 
study reported by Money, et al. [16] where, when only bilateral LSCC were inactivated, no horizontal component of PAN was observed but the vertical and torsional components were still present.

\section{"Light debris" theory}

It has been suggested that "light debris" are attached on the LSCC cupula and make the cupula "light" based on the following observations; 1) only the horizontal component of DCPN is induced in most light cupula patients, 2) positional vertigo shows abrupt onset, and 3) usually only one side is affected $[4,5,8,9]$. Although this "light debris" has not been identified as yet, several candidates have been proposed; 1) freefloating cells within the endolymph degenerate and swell up to become lighter [8], or 2) the attached otolith particles expand and becomes lighter (because apogeotropic DCPN is converted to geotropic DCPN in some patients) [8]. Ichijo [9] demonstrated that the angle of the neutral position was greater in light cupula than in heavy cupula, and speculated that light debris more commonly adheres to the canal side of the LSCC than to the utricular side, considering the microanatomy of the cupula and the cilia of the hair cells. However, this theory has been challenged based on studies in which attempts to detach any "light debris" from the cupula were not successful [7].

\section{Light cupula with or without another inner ear comorbidity}

In 1957, Aschan and Stahle [26] described three patients (from among 21 patients with Meniere's disease) showing persistent geotropic DCPN during acute attacks of vertigo and postulated that the mechanism for geotropic DCPN in Meniere's disease was similar to that in PAN. Though a null plane was not sought to be identified in the study, we can assume that a null plane might have been identified on the unaffected side from the nystagmography results in all 3 patients [26]. Patients with sudden sensorineural hearing loss $[2,4,19,21,24,27]$ and acute otitis media complicated by serous labyrinthitis [28] may exhibit persistent geotropic DCPN. Compared to patients with light cupula without associated labyrinthine disorders, a null plane may not be identified in patients with such association on the same side with the inner ear disorder, and the conversion from geotropic to apogeotropic nystagmus may occur more frequently in the latter group $[22,28]$.

\section{Treatment of Light Cupula}

Because the pathophysiological mechanism of light cupula is not known, a treatment protocol has not been established yet. Canalith repositioning procedures such as Barbecue and Gufoni maneuvers have been ineffective in treating light cupula $[2,7,15,29]$. Cha, et al. [30] reported a patient with light cupula whose positional nystagmus disappeared, though the mechanism could not be explained clearly, immediately after transcutaneous vagus nerve stimulation but reappeared afterward.

Symptoms of vertigo and positional nystagmus resolve spontaneously within 2 weeks in most patients with light cupula $[3,8]$. However, in some cases in whom persistent geotropic DCPN is accompanied by sudden sensorineural hearing loss, positional vertigo may persist for several months or even longer [19,27], and surgery such as LSCC plugging may be required in some cases, to treat intractable positional vertigo [19].

\section{Conclusion}

The light cupula has possibly been underdiagnosed thus far because the differentiation between LSCC canalolithiasis and light cupula can be challenging unless positional nystagmus is observed for a prolonged duration. When a HRT reveals persistent geotropic DCPN, and a null plane is identified, the diagnosis of light cupula can be made. The pathophysiological mechanism underlying light cupula needs to be elucidated to establish a proper treatment protocol.

\section{Acknowledgments}

This work was supported by the National Research Foundation of Korea (NRF) grant funded by the Korea government (MSIP) (2015 R1C1A1A01055849).

\section{Conflicts of interest}

The authors have no financial conflicts of interest.

\section{REFERENCES}

1) Hiruma $K$, Numata T. Positional nystagmus showing neutral points. ORL J Otorhinolaryngol Relat Spec 2004;66:46-50.

2) Hiruma $K$, Numata $T$, Mitsuhashi $T$, Tomemori $T$, Watanabe $R$, Okamoto Y. Two types of direction-changing positional nystagmus with neutral points. Auris Nasus Larynx 2011;38:46-51.

3) $\mathrm{Kim} \mathrm{CH}$, Kim MB, Ban JH. Persistent geotropic direction-changing positional nystagmus with a null plane: the light cupula. Laryngoscope 2014;124:E15-9.

4) Bergenius J, Tomanovic T. Persistent geotropic nystagmus--a different kind of cupular pathology and its localizing signs. Acta Otolaryngol 2006;126:698-704.

5) Imai T, Matsuda K, Takeda N, Uno A, Kitahara T, Horii A, et al. Light cupula: the pathophysiological basis of persistent geotropic positional nystagmus. BMJ Open 2015;5:e006607.

6) Kim CH, Shin JE, Kim YW. A new method for evaluating lateral semicircular canal cupulopathy. Laryngoscope 2015;125:1921-5.

7) Tomanovic T, Bergenius J. Vestibular findings in patients with persistent geotropic positional nystagmus: the 'light cupula' phenome- 
non. Acta Otolaryngol 2014;134:904-14.

8) Ichijo H. Persistent direction-changing geotropic positional nystagmus. Eur Arch Otorhinolaryngol 2012;269:747-51.

9) Ichijo $H$. Neutral position of persistent direction-changing positional nystagmus. Eur Arch Otorhinolaryngol 2016;273:311-6.

10) von Brevern $M$, Radtke A, Clarke AH, Lempert T. Migrainous vertigo presenting as episodic positional vertigo. Neurology 2004;62:46972.

11) Polensek SH, Tusa RJ. Nystagmus during attacks of vestibular migraine: an aid in diagnosis. Audiol Neurootol 2010;15:241-6.

12) Lechner C, Taylor RL, Todd C, Macdougall H, Yavor R, Halmagyi $\mathrm{GM}$, et al. Causes and characteristics of horizontal positional nystagmus. J Neurol 2014;261:1009-17.

13) Lee HJ, Kim ES, Kim M, Chu H, Ma HI, Lee JS, et al. Isolated horizontal positional nystagmus from a posterior fossa lesion. Ann Neurol 2014;76:905-10.

14) Yang TH, Oh SY. Geotropic central paroxysmal positional nystagmus in a patient with human immunodeficiency virus encephalopathy. J Neuroophthalmol 2014;34:159-61.

15) Choi JY, Lee ES, Kim HJ, Kim JS. Persistent geotropic positional nystagmus after meningitis: evidence for light cupula. J Neurol Sci 2017;379:279-80.

16) Money KE, Johnson WH, Corlett BM. Role of semicircular canals in positional alcohol nystagmus. Am J Physiol 1965;208:1065-70.

17) Money KE, Myles WS. Heavy water nystagmus and effects of alcohol. Nature 1974;247:404-5.

18) Tomanovic T, Bergenius J. Can the nystagmus pattern in patients with a 'light cupula' be reproduced in hemi-labyrinthectomized subjects during positional alcohol nystagmus 1? Acta Otolaryngol 2011; 131:929-36

19) Seo T, Saito K, Doi K. Intractable persistent direction-changing geotropic nystagmus improved by lateral semicircular canal plugging. Case Rep Otolaryngol 2015;2015:192764.
20) Shigeno K, Egami T, Sasano T. Experimental study of nystagmus induced by injecting various solutions into the middle ear cavity. Acta Otolaryngol 1989;108:31-7.

21) Kim CH, Shin JE, Yang YS, Im D. Sudden sensorineural hearing loss with positional vertigo: initial findings of positional nystagmus and hearing outcomes. Int J Audiol 2016;55:541-6.

22) Shin JE, Jeong KH, Ahn SH, Kim CH. Conversion between geotropic and apogeotropic persistent direction-changing positional nystagmus. Acta Otolaryngol 2015;135:1238-44.

23) Shim DB, Ko KM, Lee JH, Park HJ, Song MH. Natural history of horizontal canal benign paroxysmal positional vertigo is truly short. J Neurol 2015;262:74-80.

24) Kim CH, Choi JM, Jung HV, Park HJ, Shin JE. Sudden sensorineural hearing loss with simultaneous positional vertigo showing persistent geotropic direction-changing positional nystagmus. Otol Neurotol 2014;35:1626-32.

25) Kim CH, Shin JE, Shin DH, Kim YW, Ban JH. "Light cupula" involving all three semicircular canals: a frequently misdiagnosed disorder. Med Hypotheses 2014;83:541-4.

26) Aschan G, Stahle J. Nystagmus in Menière's disease during attacks; a nystagmographical study. Acta Otolaryngol 1957;47:189-201.

27) Kim YW, Shin JE, Lee YS, Kim CH. Persistent positional vertigo in a patient with sudden sensorineural hearing loss: a case report. J Audiol Otol 2015;19:104-7.

28) Kim CH, Yang YS, Im D, Shin JE. Nystagmus in patients with unilateral acute otitis media complicated by serous labyrinthitis. Acta Otolaryngol 2016;136:559-63.

29) Ban JH, Kim MB, Hong SM. Immediate and short-term therapeutic results between direction-changing positional nystagmus with shortand long-duration groups. Ear Hear 2016;37:243-6.

30) Cha WW, Song K, Lee HY. Persistent geotropic direction-changing positional nystagmus treated with transcutaneous vagus nerve stimulation. Brain Stimul 2016;9:469-70. 